\title{
OUTFLOW IN THE SEYFERT GALAXY NGC 7319
}

\author{
K. AOKI AND G. KOSUGI
}

National Astronomical Observatory of Japan

2-21-1, Osawa, Mitaka, Tokyo 181-8588 Japan

M. YOSHIDA

Okayama Astrophysical Observatory

Kamogata-cho, Asakuchi-gun, Okayama 719-02 Japan

H. OHTANI

Department of Astronomy, Kyoto University

Sakyo-ku, Kyoto 606-01 Japan

AND

A.S. WILSON

Department of Astronomy, University of Maryland

College Park, MD 20742 U.S.A.

\section{Introduction}

Aoki et al. (1996) found outflowing gas in the Seyfert galaxy NGC 7319. The velocity of the outflow comes up to $500 \mathrm{~km} \mathrm{~s}^{-1}$ and its extent is $4 \mathrm{kpc}$. This outflow is one of the largest outflowing phenomenon that have ever been found in Seyfert galaxies. The radio emission found by van der Hulst \& Rots (1981) aligns the outflowing gas. It suggests the radio emission has relation to the outflow. We have made higher resolution radio imaging of NGC 7319 with VLA ${ }^{1}$ to study in detail relation of radio emission to outflowing gas. These radio images have been compared to Hubble Space Telescope $(H S T)$ archival broad-band WFPC2 image. The systemic velocity $6740 \mathrm{~km} \mathrm{~s}^{-1}$ (Aoki et al. 1996) gives a distance of 90 Mpc for NGC 7319 assuming a Hubble constant $H_{0}=75 \mathrm{~km} \mathrm{~s}^{-1} \mathrm{Mpc}^{-1}$. Thus $1^{\prime \prime}$ corresponds to $450 \mathrm{pc}$.

${ }^{1}$ The National Radio Astronomy Observatory is a facility of the National Science Foundation operated under cooperative agreement by Associated Universities, Inc. 


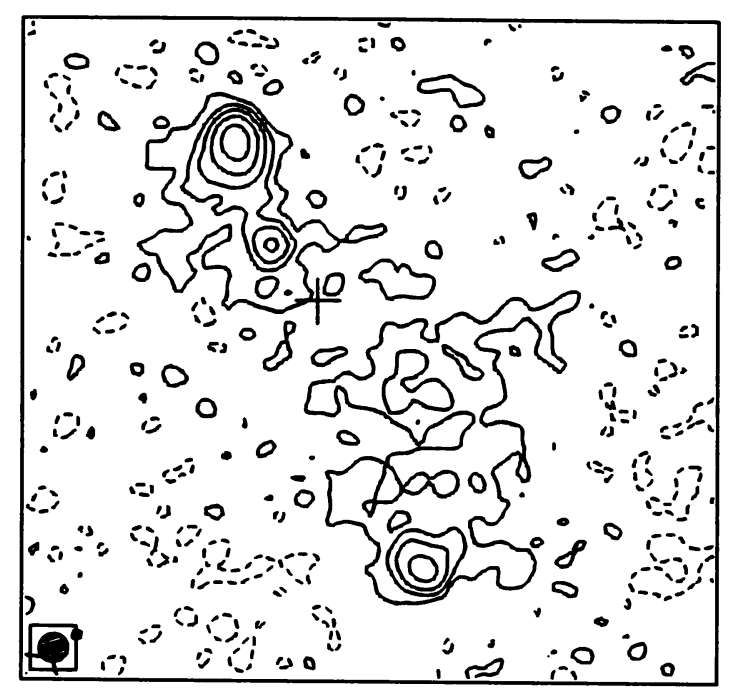

Figure 1. The contour map of the $3.6 \mathrm{~cm}$ naturally weighted image of NGC 7319. The cross indicates the optical nucleus (Clements 1983). The F.O.V. is 6." $5 \times 6 . " 5$.

\section{Results}

The VLA observations of NGC 7319 were made in the 'A' configuration at 20,6 and $3.6 \mathrm{~cm}$ on 1996 November 4 . Three compact components are clearly visible in the radio map (Fig. 1). The double or triple structure like NGC 7319 are found in many Seyfert galaxy (Ulvestad \& Wilson 1984a, $1984 \mathrm{~b}, 1989)$. The extent of radio emission is larger than $4^{\prime \prime}(2 \mathrm{kpc})$, so the size of radio emission in NGC 7319 is large one among nearby Seyferts.

We have compared our radio image to $\mathrm{F} 606 \mathrm{~W}$ image in HST archive, and found a triangular structure in $\mathrm{F} 606 \mathrm{~W}$ image at the position of the southern radio component. This structure may be a emission-line region collisional-excited by shock which a radio jet has induced. High-resolution narrow-band imaging will be needed to confirm this.

We thank AOC staffs, especially G. Taylor for their kind helps during observations and data processing.

\section{References}

Aoki, K., Ohtani, H., Yoshida, M., \& Kosugi, G. 1996, AJ, 111, 140

Clements, E. D. 1983, MNRAS, 204, 811

van der Hulst, J. M., \& Rots, A. H. 1981, AJ, 86, 1775

Ulvestad, J. S. \& Wilson, A. S. 1984a, ApJ, 278, 544

Ulvestad, J. S. \& Wilson, A. S. 1984b, ApJ, 285, 439

Ulvestad, J. S. \& Wilson, A. S. 1989, ApJ, 343, 659 